\title{
Geoinformational system of rescue services
}

\author{
Sergiy Yemelyanenko ${ }^{1, *}$, Yuriy Rudyk ${ }^{1}$, Andriy Kuzyk $^{1}$ and Roman Yakovchuk $^{1}$ \\ ${ }^{1}$ Lviv State University of Life Safety, 79007, Lviv, Ukraine
}

\begin{abstract}
Geoportal is an indispensable tool for processing data related to city infrastructure, life support systems; for the analysis of statistics of fires and other emergency situations in the city; to create thematic layers of electron-vector maps showing the city's infrastructure and other information necessary for the management of the SNS units; for identification of fire risks for residential buildings and public buildings of Lviv city and mapping them according to the risk levels in the form of a risk map. The analysis of the city's infrastructure allows us to study in detail the problematic issues related to fire and technological safety. The geoportal and its cartographic information of the information will allow the head of the rescue unit, during the post-emergency situation, to find the information necessary for the effective performance of rescue operations and to assess the potential risks.
\end{abstract}

\section{Introduction}

Electronic maps and their geographic analysis applying a variety of algorithms are increasingly using in information technology. Modern GIS technologies have the opportunity to perform not only a regular search, but also to use the mechanisms of generalization and full analysis of the information obtained when making optimal decisions based on modern approaches and means of visualization of geographical data.

These features distinguish GIS from other systems and provide unique opportunities for their use in solving various tasks related to analysis and forecasting, allocation of dominant factors, causes and possible consequences, planning of strategic and current decisions.

There are alternative noncommercial online services that offer free software [1]. Such trends indicate a mutual interest and further symbiosis of global web mapping and official structures, further institutionalization, rapid growth and consolidation, will be the second stage and most likely scenario of their development. Modernization of civil protection systems is today one of the most progressive and perspective global trends, the inclusion of which today is strategically feasible for any country in the world.

The inadequacy of existing software products and technical support in the State Emergency Service of Ukraine (SES) units that do not support the exchange and storage of map data for management decisions in emergency situations and the assessment of the effectiveness of rescue measures poses an urgent task for the development and improvement of both software products and their operation technologies. Thus, there is a need for reliable and high-quality data exchange and the application of GIS-mapping

\footnotetext{
* Corresponding author: 1dubgd@i.ua
} 
software methods and tools that can improve the efficiency of operations and rescue services and evaluate its results.

When simulating the development of emergencies, electronic and attributive information about potentially dangerous objects, forces and means for emergencies and its consequences liquidation is used. As a result of the simulation, all the initial data (places of origin of the emergencies, the dislocation of SES forces and means of liquidation of the emergencies, people living, etc.) are displayed on separate layers of the map.

The aim of the work is to improve the analytical and operative assessment of the situation of events for facilities, groups of people and local authorities, for forecasting in the short and long terms; to create a portal for analytical modules that would perform the functions of estimating and forecasting emergencies based on continuous monitoring, which would enable the developing of emergency risk maps for certain territorial units.

The article uses the results of the partnership collaboration of the scientists of Lviv State University of Life Safety (LSULS) and the private enterprise Phalcon Military Solution [2].

\section{Experimental}

\subsection{Using software for rescue service}

The proposed geoinformation system (Geoportal) consists of three software components: a cartographic editor, a program for working with maps and a monitoring server for mobile objects. Using the MicroGisEditor editor, has can create electronic vector maps and edit existing ones. The editor creates maps based on PFM - Polish Format with the ability to compile in various exchange and closed cartographic formats [3].

PFM (Polski Format Mapy) - is a text format for storing data, used as an intermediate format when converting between different industrial formats of electronic maps, and for editing them. The PFM format, also known as the "Polish format", is the source language for the GPSMapper compiler, CityGuide Constructor, 7 way and others.

Key features of the PFM format. The structure of the Polish format allows you to store cartographic data with varying degrees of detail, depending on the scale of map display. To do this, the concept of level is used. Each level has its own range of scale that does not overlap with other levels. Only one level can be displayed at a time. The Polish format can be easily expanded and supplemented with its objects or fields of objects, which makes it convenient for the development of mapping software with specific requirements. Extended Polish data formats remain compatible with programs that work with the standard Polish structure.

Geoportal provides the user with a wide range of tools for processing the database. The database can be located both in the client and in a remote location. The portal is designed to use online tools for processing information databases.

MicroGISTrack is a software, server geoinformation platform for building a satellite monitoring and monitoring system developed in accordance with international standards. The effective system of the new generation allows to carry out complex control over mobile objects by means of the Internet via information from the sensors of satellite terminals (trackers) installed on mobile objects into a single database.При наличии GPS приемника, пользователь может производить ориентирование на местности и контроль движения по маршруту.

MicroGISViewer is a geoinformation system designed to work with electronic-vector multilingual maps and raster maps prepared in MicroGISEditor, creating atlases, orienting the terrain using a GPS receiver, routing routes, tracking the route, analyzing tracks, routes, 
POIs created in navigation devices, performing various measurements and calculations, as well as applying the user a different situation.

Due to a wide range of measuring instruments (Fig. 1) in MicroGIS Viewer - the user can perform various measurements on this map.

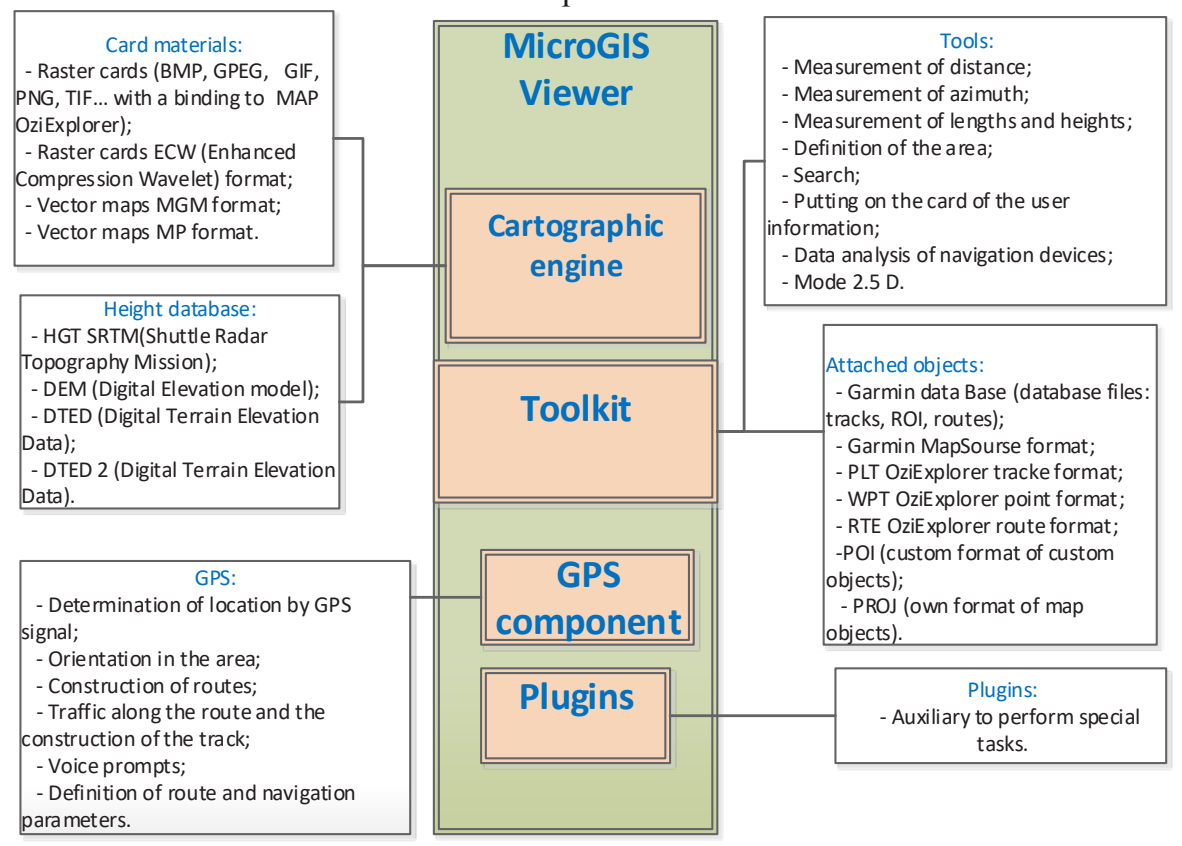

Fig. 1. MicroGIS Viewer measuring instruments.

Solved problems:

- Viewing of political, thematic, topographic, administrative, tactical, navigational vector and raster maps.

- Studying the nature of the terrain on vector, raster maps, space images.

- Study the nature of the terrain on vector and raster maps, as well as a base of heights.

- Measurement of the areas of objects on the map.

- Measurements of the lengths of objects on the map.

- Measurement of distances on the map.

- Measuring the azimuth on the map.

- Mapping of user objects (tactical situation).

- Orientation on the terrain.

- Performing various types of search for objects on the map (at the address, by type, by coordinates, by index, by phone number).

- Construction of routes on the map (on a straight line, on highways).

- Analysis of data obtained from navigation instruments GARMIN (POI, tracks, routes).

- Analysis of data created in the navigation program OziExplorer.

- Support for a variety of cartographic projections. 


\subsection{Main characteristics of the geoportal's work}

The Geoportal is a desktop that has access to modules and a large number of pages, the main module is the desktop itself, which has a substrate with remote access depending on the assignment of rights, information that is remote and user layers (Fig. 2).

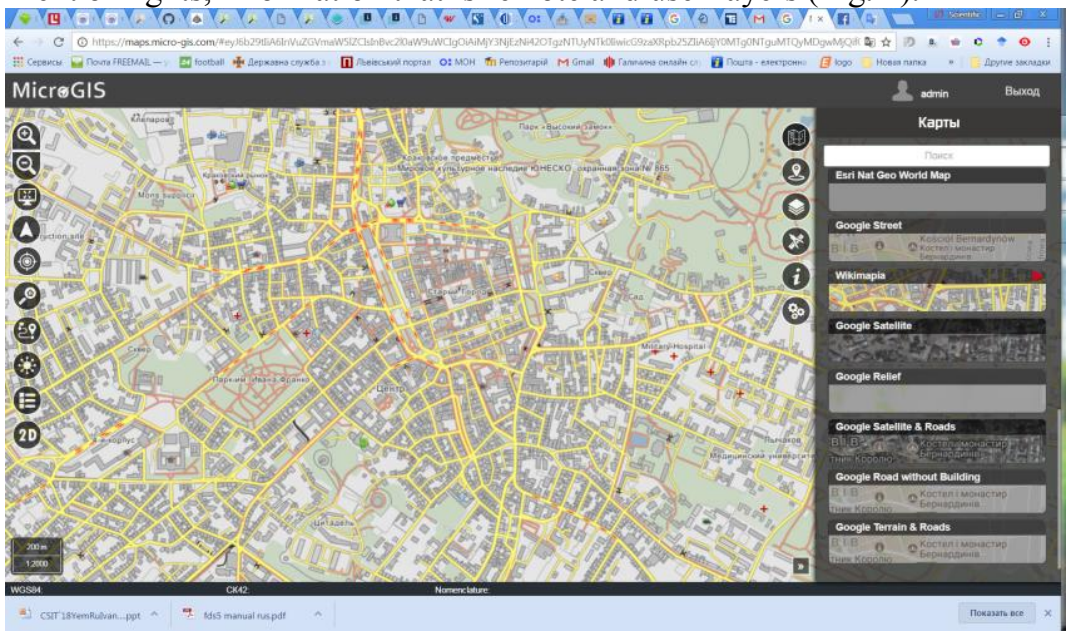

Fig. 2. Geoportal desktop.

On the basis of the software can create your own maps and use existing ones. If the user does not have his database, he can use the Geoportal database. To work the user must select the data class, the layers with which he will work or create their own layers of the map. On these layers of the map you can inflict city infrastructure objects that can help when forecasting or linking to the necessary online resources from statistics, weather conditions, and traffic. For example, to determine the individual risk of death or damage [4] with a fire in the home, the lightning injures, etc.

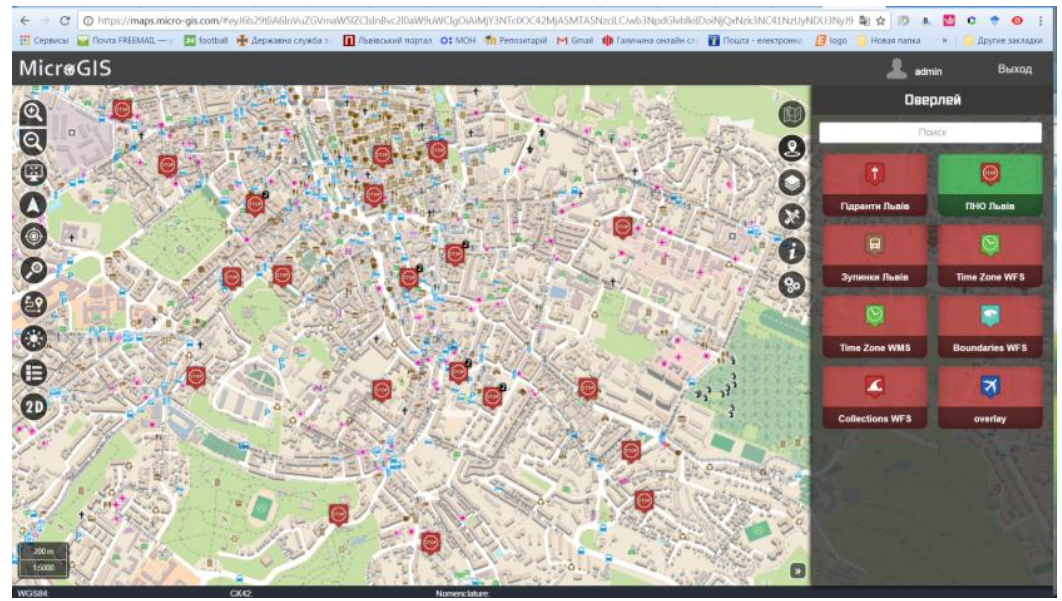

Fig. 3. Image of Geoportal with the potentially hazardous objects and other objects.

Creating the necessary maps parameters and generate a link that can be sent to the user to process the information. It is possible to put a password in order to preserve the confidentiality of information, and the link can exist for a certain period of time. 
In the Geoportal, important critical infrastructure objects can be identified. They include any place of accumulation of people, increased fire danger, bomb shelters, etc. Therefore, there is a need for a clear demarcation of access that would allow free access to general information for any user of the online resource and a request for the corresponding "key" (login and password) for more detailed information that is not intended for the public. That is, in a Geoportal, to access some of the layers requires user authorization.

Technologic safety of the city Lviv is conditioned by the placement on its territory of 245 potentially hazardous objects ( $\mathrm{PHO}$ ) and objects of high danger (OHD), of which OHD 1 class - 52, OHD 2 class - 62, the remaining 131 - PHO. These objects contain a large number of potent poisonous, explosive and fire hazard substances [5].

It is necessary to mitigate the consequences of the influence of the emergencies of manmade origin due to increased efficiency and effectiveness of the response of special services in emergencies through cartography, the accumulation of sufficient material and financial resources and information data collection to eliminate such situations. The implementation of this will reduce the number of emergencies by $5-7 \%$, the number of dead - 5-6, injured $3-5$, the magnitude of material damage - by $2-4 \%$ annually [6].

\section{Results and discussion}

With this software you can create your own maps and use existing ones. These maps can be applied to city infrastructure objects that can help with prediction or elimination of emergencies. In particular, the mapping of hydrants, reservoirs, critical infrastructure objects and units of the SES of Ukraine provides assistance in rapid orientation and response during emergencies and the extinguishing of fires of various classes.

\subsection{Key features for operational activities}

The cartographic image will allow the head of fire and rescue unit to determine the places for water intake, and identify the group of dwelling houses through the graphical interface of the Geoportal. Determine if there is a possibility of spreading an emergency to other objects, including critical infrastructure objects. The Geoportal has the ability to work with a large number of open mapping services: Google, Yandex, OSM, WikiMapia and others. This allows determining online traffic congestion, the optimal and shortest route. With the help of the tracker located on a fire and rescue vehicle, the dispatcher can monitor the movement of the unit and use Geoportal to communicate online, and the camera on an operational vehicle allows taking a photo of the operational situation during an emergency and transferring the image to the dispatcher.

So the dispatcher can log in and track the movement of the fire truck, coordinate it with the road map, on a less loaded route (which is very significant at rush hours in large cities), and what is important is to reduce the time of arrival to the location.

Using the Geoportal provides the opportunity to create a database of objects in the form of thematic maps on the background of a cartographic basis, in particular: linear, plane, point, 3D models of buildings and territories. By objects (for example: a high-rise building, a building with a massive presence of people, a potentially hazardous object, an object of increased hazard, etc.) information of any type (fire cards, layouts, operational and tactical characteristics of buildings) can be fixed [7,8]. It can be textual, graphic, video and audio. $3 \mathrm{D}$ terrain modeling allows you to virtually inspect an object. This assumes that for the monitoring of a fire truck, the dispatcher must enter the number of his part, as well as the number of the car that went on the call. In turn, this activates the connection to the Geoportal data obtained from the GPS navigator located in the car. 
If a regular user has visited the Geoportal (without his right to access classified information), he will only be able to view some of the layers of the maps, such as: gas stations, underground passages, new buildings, historic buildings and monuments, and also plans to connect the maps routes and stops with tracking of the current location of all types of public transport. And what is very important is a map of the location of shelters, medical facilities, and SES units. Thus, for viewing, those layers will be available, which at the same time contain useful information that may be vital in some situations in the emergency situations, and in everyday life, but does not cover information that could be useful to any kind of criminals, or whatever they are could not find it in free access.

\subsection{Use of analytical modules}

The Geoportal supports various analytical modules, which can be used to: calculate the fire risk of objects, to predict possible hazardous areas of chemical hazardous substances on objects of high hazard, etc.

Corresponding software can be connected to the Geoportal to calculate and predict the area of damage during the release of hazardous substances $[9,10]$. Calculation will be done online, which means that it will be taken into account except for the consistency, mass and type of substance, and also weather conditions (temperature, humidity, direction and velocity of wind gusts and atmospheric pressure) (fig. 4).

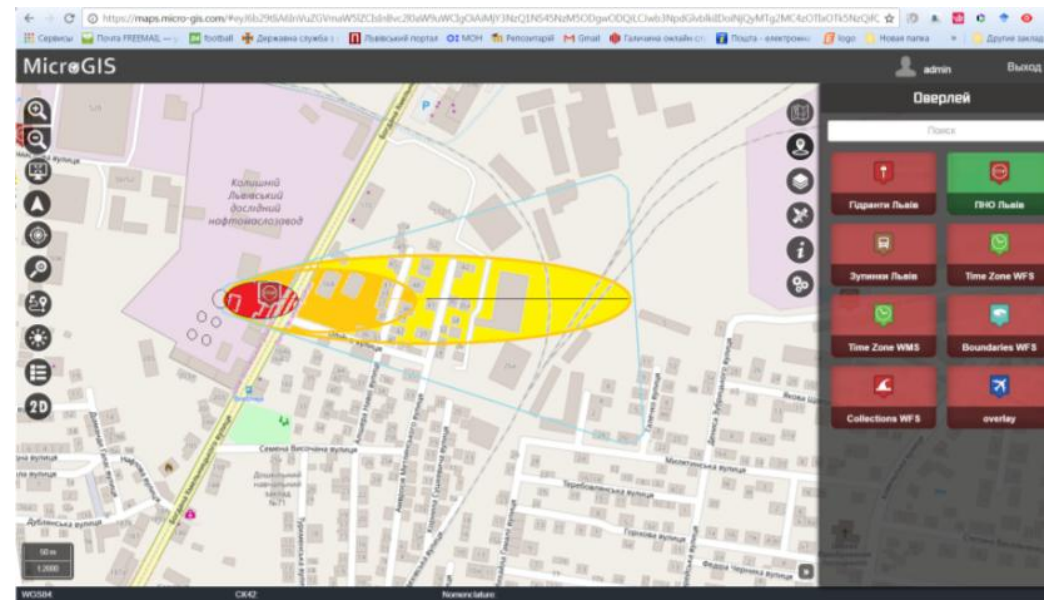

Fig. 4. Prediction of possible hazardous areas of chemical-dangerous substances at high-risk objects.

This will help predict precisely the area of the damage, and pre-create a zone of evacuation of the population. This in turn will reduce the number of injuries, injuries and civilian casualties.

Analytical module for forecasting the risk of death from fire in residential buildings and public buildings of Lviv. Forecasting of fire risks in residential buildings is realized on the basis of the proposed method of calculation of fire risks [1]. Making a risk card die from a fire in the appropriate group of the house for a period of 10 years in the corresponding colors allows you to build a map of the risk to know the potential risks and hazards of the objects.

Also, the Geoportal can aggregate of fire parts forms an irregular network. We set the task of constructing a regular network for the function of the density of fire cells with a sufficiently small cell size (square grid), which is necessary for mapping this parameter. For this purpose, a secondary regular square grid is constructed with a side of $1 \mathrm{~km}$. It is 
necessary to determine the value of the density function in each node of this grid. The problem is solved by the method of inverse weighted distances using Voronoi diagrams [11-13].

For the transition from the irregular to the regular model of the density of the location of the fire and rescue parts, the triangulation of Delone [14] was used.

Triangulation is a triangular polygonal network formed on a plurality of point objects by connecting them to segments that do not intersect. At the heart of the triangulation of Delaunus is a circular criterion: if you draw a circle through arbitrary three points, then the other points should not fall into it, with all the triangles of the network tend to the correct form.

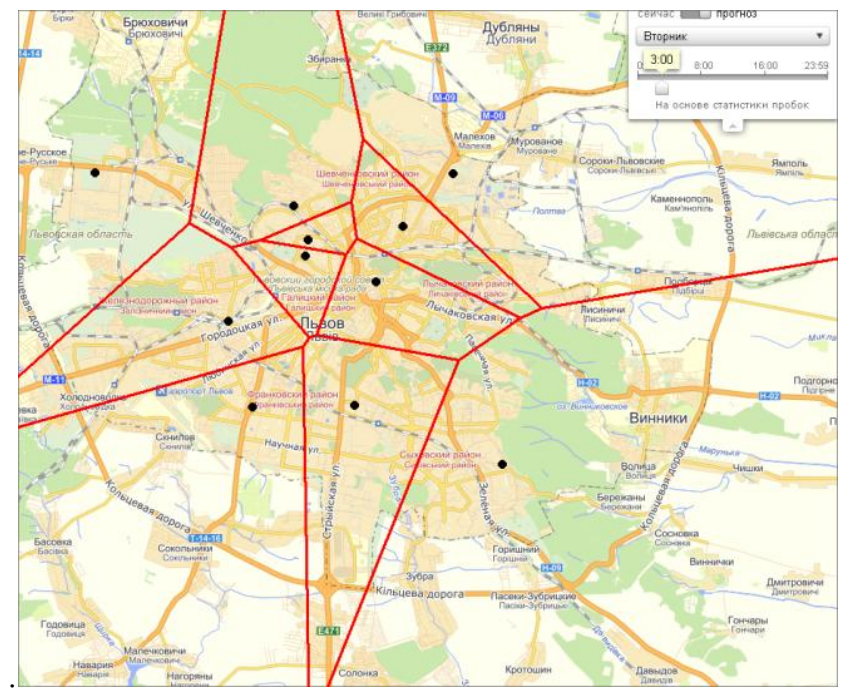

Fig. 5. Distribution of fire depots for uniform density.

Also, the Geoportal can monitor the tendency of fires in certain areas of the city, bringing all calls to the corresponding database, and displaying everyone on the map. According to this map, monitoring of the emergency situations can be carried out, and conclusions can be drawn from the leadership on increasing the cost of anti-fire means and advocating the fight against the causes of the emergency situations in the area and increasing the number of inspections of premises, both residential and industrial, to comply with fire safety norms.

The database may also include objects that are potentially dangerous in the event of unpredictable weather conditions, such as exceeding the rate of precipitation several times, which will entail the flooding of many objects and buildings, such as underground transitions. Again, according to the forecasts of weather forecasters, it is possible to prepare the equipment or to warn the appropriate emergency situations in place.

\section{Conclusions}

1. Geoinformation complex is an auxiliary tool for rescuer in making management decisions during the elimination of fires or other emergencies.

2. With the help of additional modules, such as fire risk assessment, it is possible to predict the possibility of occurrence of emergency situations, both at the stages of operation and design.

3. With the help of the program, it is possible to predict possible accidents on objects of high danger and potentially hazardous objects with chemical-dangerous substances. 
4. Using the Voronoi charts, you can evenly distribute the rescue vehicle exit zone.

5. The geoportal and its cartographic information allow the rescue unit manager during an emergency to find the necessary information for the effective conduct of rescue operations and to assess potential risks.

6. Sergiy Yemelyanenko and Yuriy Rudyk express their gratitude to State Emergency Service of Ukraine for the opportunity to participate in Project No 0114 U004182.

\section{References}

1. S. Yemelyanenko, Yu. Rudyk, A. Ivanusa, Geoinformational system for risk assessment visualization, Proceedings of the XIIth International Scientific and Technical Conference CSIT 2018, Lviv Polytechnic National University, 17 (2018)

2. Geoinformation software complex MicroGIS [Electronic resource]. - Access mode: http://www.micro-gis.com/index.php/main.html

3. MicroGisEditor, Access mode: http://www.micro-gis.com/index.php/editor.html

4. S. Jonkman, J. Hazard. Mater. A99, 1 (2002)

5. S. Yemelyanenko, A. Kuzyk, Estimation of the time of the fire and rescue units' followup to the place of fire (on the example of the city of Lviv) Bulletin of the commandengineering institute of the Ministry of Emergencies of the Republic of Belarus, Minsk 1, 24 (2016)

6. C. Bouchard, In the United States: a reference work on the Nation's fire problem (Nova Science Publishers, New York, 2010)

7. S. Gnatjuk, Crisis Mapping: Modern online technology for the prevention and response of emergencies Strategic priorities, 3 (2012) [Electronic resource] Access mode :http://www.niss.gov.ua/public/File/Str_prioritetu/SP_3_2012.pdf

8. V.K. Dolja, P.M. Gritsyuk, M.Ye. Kristopchuk, Investigation of the transport network of the region by the method of constructing the function density of the population, municipal economy of cities (Kharkiv National University of Urban Management named after A. Beketov, 69, 205, 2006)

9. J. Fitch, E. Raber, D. Imbro, Sci. 302/5649, 1350 (2003)

10. ALOHA [Electronic resource]. - Access mode: https://response.restoration.noaa.gov/sites/default/files/aloha.pdf

11. G. Voronoi, Nouvelles applications des parameters continues à la therie des formes quadratiques Deuxième Mémorie: Recherches sur les parralléloèddres primitifs J. reine angew. Math. 134, 198 (1908)

12. A. Kuzyk, O. Karabyn, O. Trusevych, Analysis of areas of service of fire and rescue parts using Voronoi diagrams Fire safety: Collection of scientific works, LDUBZD 13, $73(2008)$

13. A. Okabe, B. Boots, K. Sugihara, S.N. Chiu, D. Kendall, Spatial Tesselletions: Concepts and Applications of Voronoi Diagrams (New York, 2000)

14. D. Watson, Comp. J. 24/2, 167 (1981) 